\title{
Aplicação de adubo e corretivo após o corte da cana-planta utilizando técnicas geoestatísticas
}

\author{
Fertilizer and limestone applications after the harvest of sugarcane as determined by \\ geostatistics techniques
}

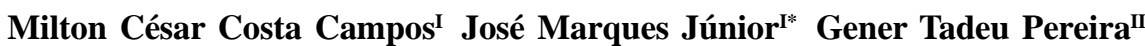 \\ Zigomar Menezes de Souza ${ }^{\text {III }}$ Diogo Mazza Barbieri ${ }^{I}$
}

\section{RESUMO}

O conhecimento da variação espacial de atributos do solo pode contribuir para o planejamento e a otimização na condução de experimentos e de lavouras comerciais, objetivando a agricultura de precisão. O objetivo deste trabalho foi avaliar a necessidade de fertilizante e calcário para implantação da agricultura de precisão, com base no comportamento espacial de atributos químicos de um Latossolo após o corte da cana-planta. Os dados foram avaliados pela estatística descritiva e análise da dependência espacial, com base no ajuste de semivariogramas. As técnicas de geoestatísticas apresentam-se como ferramentas auxiliares na aplicação de fertilizantes por meio de taxa variada, detectando, para a área em estudo, que não se recomenda a aplicação de fósforo $e$ calagem por taxa variada.

Palavras-chave: agricultura de precisão, variabilidade espacial, atributos químicos.

\section{ABSTRACT}

Knowing the spatial variation of soil properties can contribute for planning and optimization of experiments and commercial crops based on precision agriculture. The objective of this research was to evaluate the necessity of fertilizer and limestone applications for the implantation of precision agriculture techniques based on the spatial behavior of chemical attributes of one Latosol after the harvest of the sugarcane. The data were evaluated by means of descriptive statistics and spatial dependence analysis with the adjustment of semivariograms. The geostatistics techniques applied were found to be suitable as auxiliary tools in the fertilizer application by means of variable rates. It was detected that phosphorus and limestone applications were not necessary according to the variable rate.

Key words: precision agriculture, spatial variability, chemical attributes.

\section{INTRODUÇÃO}

Com o aumento das práticas de mecanização agrícola e a exigência da produção em larga escala, os campos cultivados normalmente têm sido tratados de forma uniforme. Portanto, as taxas de aplicação de fertilizantes são calculadas com base em valores médios, e a aplicação é feita uniformemente em toda a extensão do campo, porém, este tratamento pode gerar perdas econômicas ao produtor e provocar danos ambientais (SPAROVEK \& SCHNUG 2001).

Solos de mesma classe, quando submetidos a manejos diferenciados, podem apresentar diferenças significativas em seus atributos no espaço (BOUMA et al.,1999). No Brasil, grandes áreas agrícolas sobre Latossolos, considerados homogêneos do ponto de vista pedológico, apresentam diferenças na distribuição espacial de seus atributos de acordo com o manejo adotado ao longo de sucessivos cultivos (MOTOMIYA et al., 2006).

Buscando resolver essa problemática, têmse aplicado algumas tecnologias ligadas à agricultura de precisão, cuja finalidade é produzir ferramentas para aumentar a eficiência no gerenciamento da agricultura, permitindo, assim, a aplicação de insumos nos locais corretos e nas quantidades requeridas (MZUKU et al., 2005; DERCON et al., 2006). Dentre essas novas tecnologias, a geoestatística surge como uma das técnicas mais importantes, visto que ela poderá propiciar a redução dos custos e o aumento de

IPrograma de Pós-graduação em Ciência do Solo, Departamento de Solos e Adubos, Faculdade de Ciências Agrárias e Veterinárias (FCAV), Universidade Estadual de São Paulo (UNESP), 14870-900, Jaboticabal, SP, Brasil. E-mail: marques@fcav.unesp.br. *Autor para correspondência

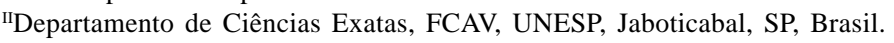

IIIUniversidade Estadual de Campinas (UNICAMP), Faculdade de Engenharia Agrícola, Campinas, SP, Brasil. 
produtividade das culturas, principalmente quando aplicada a culturas economicamente expressivas, como a cana-de-açúcar (JOHNSON \& RICHARD JÚNIOR, 2005).

Este conceito é evidenciado pelo trabalho de BORGELT et al. (1994), o qual, depois de amostrar o pH do solo de uma área de 8,8ha, concluiu que sem a utilização da técnica de aplicação localizada 9 a $12 \%$ dessa área receberia uma quantidade de calcário acima da recomendada, enquanto que 37 a $41 \%$ receberia uma quantidade abaixo da recomendada. CORÁ \& BERALDO (2006), ao estudar variabilidade espacial de atributos do solo antes e após calagem e fosfatagem em doses variadas na cultura de cana-de-açúcar, observaram que os mapas de isolinhas elaborados por meio da krigagem apresentaram maior precisão e diferentes padrões de ocorrência da distribuição espacial dos atributos, comparado aos mapas de isolinhas construídos utilizando-se o interpolador linear para estimativa dos valores em locais não medidos.

O objetivo deste trabalho foi avaliar a necessidade de fertilizante e calcário para implantação da agricultura de precisão, a partir do comportamento espacial de atributos químicos de um Latossolo após o corte da cana-planta.

\section{MATERIAL E MÉTODOS}

A área de estudo localiza-se no nordeste do Estado de São Paulo, no município de Pereira Barreto, SP, $20^{\circ} 41^{\prime} 15^{\prime \prime}$ de latitude sul e $51^{\circ} 03^{\prime} 45^{\prime \prime}$ de longitude oeste, na província geomorfológica do Planalto Ocidental Paulista. O relevo é predominantemente plano a suave ondulado, com declividades média variando de 3 a 5\% e altitude oscilando entre 335 a 390m acima do nível do mar. Nesta região predominam os Latossolos, distribuídos ao longo da vertente, com perfis retilíneos e convexos. Nas posições mais elevadas e planas, encontram-se o Latossolo Vermelho distroférrico textura média, cujo material de origem derivou-se principalmente de arenitos da Formação Santo Anastácio, e estes transicionam encosta abaixo para Latossolo Vermelho eutroférrico textura argilosa, originado principalmente dos produtos da alteração dos basaltos Formação Serra Geral (IPT, 1981). O clima da região, segundo a classificação de Köppen, é do tipo tropical chuvoso de bosque com chuvas de verão e estiagem no inverno (Aw). A área de estudo é atualmente cultivada com cana-de-açúcar, que foi implantada em março de 2004, em área de pastagem.

A amostragem do solo foi realizada em uma área de 505ha, dividida numa malha com espaçamento regular de 50m entre pontos, sendo uma amostragem a cada 7ha, perfazendo o total de 72 pontos. De acordo com a fertilidade do solo e a disponibilidade de torta de filtro na usina, foram utilizadas três recomendações para a adubação e a correção do solo por ocasião da implantação, utilizando-se a fórmula 10-30-20 na forma sólida para a adubação de plantio e calcário dolomítico para correção do solo: $500 \mathrm{~kg} \mathrm{ha}^{-1}$ e 2t ha-1 (em 90ha), $550 \mathrm{~kg} \mathrm{ha}^{-1}$ e $3 \mathrm{t} \mathrm{ha}^{-1}$ (em 156ha) e, 600 kg ha-1 e 4t ha-1 (em 259ha). Para adubação de cobertura, utilizou-se 250L ha $^{-1}$ de torta de filtro na forma líquida em 285ha e 200L ha ${ }^{-1}$ no restante da área.

Após a colheita da cana-planta, em julho de 2005, foi realizado um novo mapeamento da área (505 ha), utilizando-se equipamento de sistema de posicionamento global (GPS). Em cada ponto de amostragem, foram coletadas aleatoriamente, com trado tipo holandês, 5 subamostras de solo na entrelinha, num raio de $2 \mathrm{~m}$ do ponto central. Tal procedimento foi adotado para compor uma amostra composta representativa do ponto de amostragem. A intensidade de amostragem igual a uma amostra por 7 hectares foi definida baseando-se no que é praticado pela usina, baseado em pesquisas para implantação de agricultura de precisão.

Na caracterização química do solo, foram determinados $\mathrm{pH}$ em $\mathrm{CaCl}_{2}$ e acidez potencial $\left(\mathrm{H}^{+}+\mathrm{Al}^{3+}\right)$, as bases trocáveis cálcio $\left(\mathrm{Ca}^{2+}\right)$, magnésio $\left(\mathrm{Mg}^{2+}\right)$ e potássio $\left(\mathrm{K}^{+}\right)$, segundo RAIJ et al. (2001). Por fim, foram calculados os valores da soma de bases (SB), de capacidade de troca catiônica à pH 7,0 (CTC) e de saturação por bases (V\%).

Para o cálculo da necessidade de calagem e de adubação com fósforo e potássio na área, foi considerada a variabilidade espacial e, posteriormente, foi calculada a porcentagem de área de cada classe de aplicação dos insumos em estudo. Para o cálculo da necessidade de corretivo, utilizou-se o método da saturação por bases (RAIJ et al., 1997), na qual leva-se em consideração o V\% atual do solo, a CTC e o nível de saturação por bases ideal para cultura, que no caso da cana-de-açúcar é de 60\%. Para o cálculo da dose, o PRNT do calcário foi considerado $100 \%$. A adubação $\left(\mathrm{kg} \mathrm{ha}^{-1}\right)$ de fósforo $\left(\mathrm{P}_{2} \mathrm{O}_{5}\right)$ e potássio (K2O) foi baseada na tabela de adubação propostas por RAIJ et al. (1997) com produtividade esperada de 100 a 150 t ha $^{-1}$ para cana-planta após o corte.

Os atributos químicos e a necessidade de adubação e calagem foram analisados por meio da análise estatística descritiva calculando-se a média, mediana, desvio padrão, variância, coeficiente de variação, coeficiente de assimetria e coeficiente de curtose. A hipótese de normalidade dos dados foi 
testada pelo teste de Kolmogorov-Smirnov, por meio do programa computacional SAS (SCHLOTZHAVER \& LITTELL, 1997).

A dependência espacial foi analisada por meio de ajustes de semivariogramas (VIEIRA et al., 1983), com base na pressuposição de estacionariedade da hipótese intrínseca, a qual é estimada por:

$\hat{\gamma}(h)=\frac{1}{2 N(h)} \sum_{i=1}^{N(h)}\left[Z\left(x_{i}\right)-Z\left(x_{i}+h\right)\right]^{2}$ em que $\mathrm{N}$ (h) é o número de pares experimentais de observações $\mathrm{Z}\left(\mathrm{x}_{\mathrm{i}}\right)$ e $\mathrm{Z}\left(\mathrm{x}_{\mathrm{i}}+\mathrm{h}\right)$ separados por uma distância h. O semivariograma é representado pelo gráfico $\hat{\gamma}(h)$ versus h. Do ajuste de um modelo matemático aos valores calculados de $\hat{\gamma}(h)$, são estimados os coeficientes do modelo teórico para o semivariograma (o efeito pepita, $\mathrm{C}_{0}$; patamar, $\mathrm{C}_{0}+\mathrm{C}_{1}$; e o alcance, a). Para analisar o grau da dependência espacial dos atributos em estudo, utilizou-se a classificação de CAMBARDELLA et al. (1994), em que são considerados de dependência espacial forte os semivariogramas que têm um efeito pepita $<25 \%$ do patamar, moderada quando está entre 25 e $75 \%$, e fraca $>75 \%$.

Os modelos de semivariogramas considerados foram o esférico, o exponencial, o linear e o gaussiano, sendo ajustados por meio do programa $\mathrm{GS}^{+}$e posteriormente tais modelos foram usados no desenvolvimento de mapas de isolinhas (krigagem). Em caso de dúvida entre mais de um modelo para o mesmo semivariograma, considerou-se o maior valor do coeficiente de correlação obtido pelo método de validação cruzada e menor SQR (soma de quadrados do resíduo). Para elaboração dos mapas de distribuição espacial das variáveis, foi utilizado o programa Surfer (GOLDEN SOFTWARE, 1999). Os mapas gerados no
Surfer foram exportados para o programa Idrisi Kilimanjaro, onde foram calculadas as áreas que cada classe do atributo estudado tinha em relação à área total.

\section{RESULTADOS E DISCUSSÃO}

Os resultados da estatística descritiva para os atributos químicos são apresentados na tabela 1 . Verifica-se, por meio do teste Kolmogorov-Smirnov, normalidade para o potássio e a saturação por bases. De acordo com ISAAKS \& SRIVASTAVA (1989), o coeficiente de assimetria é mais sensível a valores extremos do que a média e o desvio padrão, uma vez que um único valor pode influenciar fortemente 0 coeficiente de assimetria, pois os desvios entre cada valor e a média são elevados à terceira potência. Para o presente trabalho, foram encontrados valores próximos a zero de assimetria para todos os atributos estudados, com exceção para as variáveis fósforo e necessidade de fósforo (Tabela 1).

Os valores das medidas de tendência central (média e mediana) são próximos para as variáveis potássio e saturação por bases e coeficiente de assimetria e curtose próximos de zero (Tabela 1), mostrando padrão de distribuição simétrico, não ocorrendo para as demais variáveis. Segundo ISAAKS \& SRIVASTAVA (1989), mais importante que a normalidade dos dados é a ocorrência do efeito proporcional em que a média e a variância dos dados não sejam constantes na área de estudo. Este fato não ocorreu, visto que os semivariogramas apresentaram patamares bem definidos.

Pelos limites de coeficiente de variação propostos por WARRICK \& NIELSEN (1980) para a classificação de variáveis do solo (CV < 12\%), (12\% < $\mathrm{CV}>24 \%)$ e $(\mathrm{CV}>24 \%)$ para baixa, média e alta

Tabela 1 - Estatística descritiva para as variáveis fósforo $-\mathrm{P}\left(\mathrm{mg} \mathrm{dm}^{-3}\right)$, potássio $-\mathrm{K}\left(\mathrm{mmol}_{\mathrm{c}} \mathrm{dm}^{-3}\right)$, capacidade de troca de cátions $-\mathrm{CTC}$ $\left(\mathrm{mmol}_{\mathrm{c}} \mathrm{dm}^{-3}\right)$ saturação de bases - V\%, necessidade de fósforo - NP (kg ha $\left.{ }^{-1}\right)$, necessidade de potássio - NK $\left(\mathrm{kg}\right.$ ha $\left.{ }^{-1}\right)$ e necessidade de calagem - NC $\left(\mathrm{t} \mathrm{ha}^{-1}\right)$, de amostras coletadas na profundidade de $0,0-0,2 \mathrm{~m}$, em amostragem de solo após o corte da cana-planta.

\begin{tabular}{lllllll}
\hline Estatística descritiva & $\mathrm{P}$ & $\mathrm{K}$ & $\mathrm{CTC}$ & $\mathrm{V} \%$ & $\mathrm{NP}$ & $\mathrm{NK}$ \\
\hline Média & 3,90 & 2,18 & 54,46 & 52,58 & 174,60 & 88,80 \\
Mediana & 4,00 & 2,20 & 52,00 & 53,05 & 178,00 & 82,00 \\
${ }^{1}$ DP & 1,45 & 0,98 & 21,47 & 10,95 & 17,26 & 28,15 \\
Variância & 1,60 & 0,97 & 464,50 & 119,92 & 298,00 & 792,49 \\
${ }^{2}$ CV (\%) & 37,32 & 44,95 & 39,42 & 20,82 & 9,88 & 0,53 \\
Assimetria & 1,56 & 0,11 & 0,69 & 0,12 & $-2,94$ & $0,31,70$ \\
Curtose & 4,06 & $-0,06$ & $-0,73$ & $-0,30$ & 6,85 & 0,13 \\
${ }^{3} \mathrm{~d}$ & 0,24 & $0,09^{\text {ns }}$ & 0,16 & $0,08^{\mathrm{ns}}$ & 0,33 & 0,25 \\
\hline
\end{tabular}

${ }^{1} \mathrm{DP}=$ desvio padrão; ${ }^{2} \mathrm{CV}=$ coeficiente de variação; ${ }^{3} \mathrm{~d}=$ teste de normalidade, ${ }^{\mathrm{ns}}$ não significativo pelo teste de Kolmogorov-Smirnov. 
variabilidade, respectivamente, apenas a necessidade de fósforo apresentou baixa variabilidade (9,88\%), pois a ampla faixa de comparação dos teores de fósforo promove uma pequena variação para recomendação deste atributo. A saturação por bases apresentou média variabilidade (20,82\%), sendo que as demais variáveis apresentaram alta variabilidade (Tabela 1). Resultados semelhantes foram observados por CORÁ \& BERALDO (2006), que encontraram média variabilidade para saturação de bases e baixa para a necessidade de fosfatagem, ao estudarem a variabilidade espacial de atributos de solo antes e após a calagem e fosfatagem na cultura de cana-de-açúcar.

Um coeficiente de variação maior que 35\% revela que a série é heterogênea e a média tem pouco significado. Se for maior que $65 \%$, a série é muito heterogênea e a média não tem significado algum. Porém, se for menor que 35\%, a série é homogênea e a média tem significado, podendo ser utilizada como representativa da série de onde foi obtida (VANNI, 1998). Portanto, os atributos fósforo, potássio, CTC e necessidade de calagem apresentaram série de dados heterogêneos e a média tem pouco significado.

Os resultados da análise geoestatística mostraram que todas as variáveis analisadas apresentaram dependência espacial (Tabela 2). As variáveis em estudo ajustaram-se ao modelo esférico, com exceção das variáveis fósforo e necessidade de fósforo que ajustaram ao modelo exponencial. A análise da relação $\mathrm{C}_{0} /\left(\mathrm{C}_{0}+\mathrm{C}_{1}\right)$ mostrou grau de dependência espacial forte para as variáveis capacidade de troca catiônica, saturação por bases e necessidade de calagem e grau de dependência espacial moderada para as demais variáveis em estudo (Tabela 2). Isso demonstra que os semivariogramas explicam a maior parte da variância dos dados experimentais.

O alcance indica o limite da dependência espacial da variável, ou seja, determinações realizadas a distâncias maiores que o alcance tem distribuição espacial aleatória e, por isso, são independentes entre si. Por outro lado, determinações realizadas em distâncias menores que o alcance são correlacionadas umas as outras, o que permite que se façam interpolações para espaçamentos menores que os amostrados. As variáveis em estudo podem ser divididas em dois grupos com alcance próximos. As variáveis fósforo e necessidade de fósforo apresentaram alcance variando entre 1122 e $1770 \mathrm{~m}$ (Tabela 2), para o potássio, capacidade de troca catiônica, saturação por bases, necessidade de potássio e de calagem. Os valores de alcance variaram de 2331 a 2587.

Observa-se, na figura 1, o comportamento espacial dos atributos em estudo, percebe-se que o fósforo e o potássio distribuem-se no terreno de forma mais aleatória quando comparado com a capacidade de troca catiônica e a saturação por bases, sendo que esta distribuição aleatória pode ser explicada devido ao fósforo e ao potássio serem geralmente distribuídos em linha, provocando variabilidade espacial, e devido às doses diferenciadas na implantação da área (SCHLINDWEIN \& ANGHINONI, 2000). Verifica-se, para a capacidade de troca catiônica e saturação de bases, uma tendência dos maiores valores encontraremse nos locais de menores cotas, onde ocorre presença de solos originários a partir de derrames basálticos. Segundo JUNG et al. (2006), esse fato pode estar relacionado com a posição da paisagem, que está em constante rejuvenescimento.

Observa-se que em 86\% da área os valores médios de V\% não atingiram o esperado com a calagem, ou seja, V\% igual a $60 \%$, entretanto, $40 \%$ da área ficaram próximos de $60 \%$ e $14 \%$ dentro do recomendado (Tabela 3). Porém, a necessidade de calagem obtida pela krigagem (Figura 1) variou de 0,0 a $1,5 \mathrm{t} \mathrm{ha}^{-1}$, para a aplicação de calcário a taxas variáveis, com 82\% da

Tabela 2 - Modelos e parâmetros estimados dos semivariogramas experimentais para as variáveis fósforo - P (mg dm $\left.{ }^{-3}\right)$, potássio - K (mmol $\left.\mathrm{dm}^{-3}\right)$, capacidade de troca de cátions - CTC $\left(\mathrm{mmol}_{\mathrm{c}} \mathrm{dm}^{-3}\right)$ saturação de bases - V\%, necessidade de fósforo - NP $\left(\mathrm{kg} \mathrm{ha}^{-1}\right)$, necessidade de potássio - NK $\left(\mathrm{kg} \mathrm{ha}^{-1}\right)$ e necessidade de calagem - NC $\left(\mathrm{t} \mathrm{ha}^{-1}\right)$, em amostragem de solo após o corte da canaplanta.

\begin{tabular}{lccccccc}
\hline Parâmetros & $\mathrm{P}$ & $\mathrm{K}$ & $\mathrm{CTC}$ & $\mathrm{V} \%$ & $\mathrm{NP}$ & $\mathrm{NK}$ & $\mathrm{NC}$ \\
\hline Modelo & Exponencial & Esférico & Esférico & Esférico & Exponencial & Esférico & Esférico \\
Efeito pepita $\left(\mathrm{C}_{0}\right)$ & 1,020 & 0,368 & 28,00 & 14,90 & 288 & 290 & 0,024 \\
Patamar $\left(\mathrm{C}_{0}+\mathrm{C}_{1}\right)$ & 2,041 & 1,109 & 706,9 & 109,30 & 925,20 & 925 & 0,289 \\
Alcance $(\mathrm{a})$ & 1770 & 2395 & 2587 & 2389 & 1122 & 2547 \\
${ }^{1}\left[\mathrm{C}_{0} /\left(\mathrm{C}_{0}+\mathrm{C}_{1}\right)\right] \mathrm{x} 100$ & 50 & 33 & 4 & 14 & 31 & 31 \\
${ }^{2} \mathrm{R}^{2}$ & 0,76 & 0,96 & 0,98 & 0,98 & 0,97 & 0,97 \\
${ }^{3} \mathrm{SQR}$ & 0,124 & 0,017 & 8064 & 130 & 6537 & 0,98 & 10125 \\
\hline
\end{tabular}

${ }^{1} \mathrm{RDE}$ = razão de dependência espacial; ${ }^{2} \mathrm{R}^{2}$ = coeficiente de determinação; ${ }^{3} \mathrm{SQR}$ = soma dos quadrados dos resíduos. 


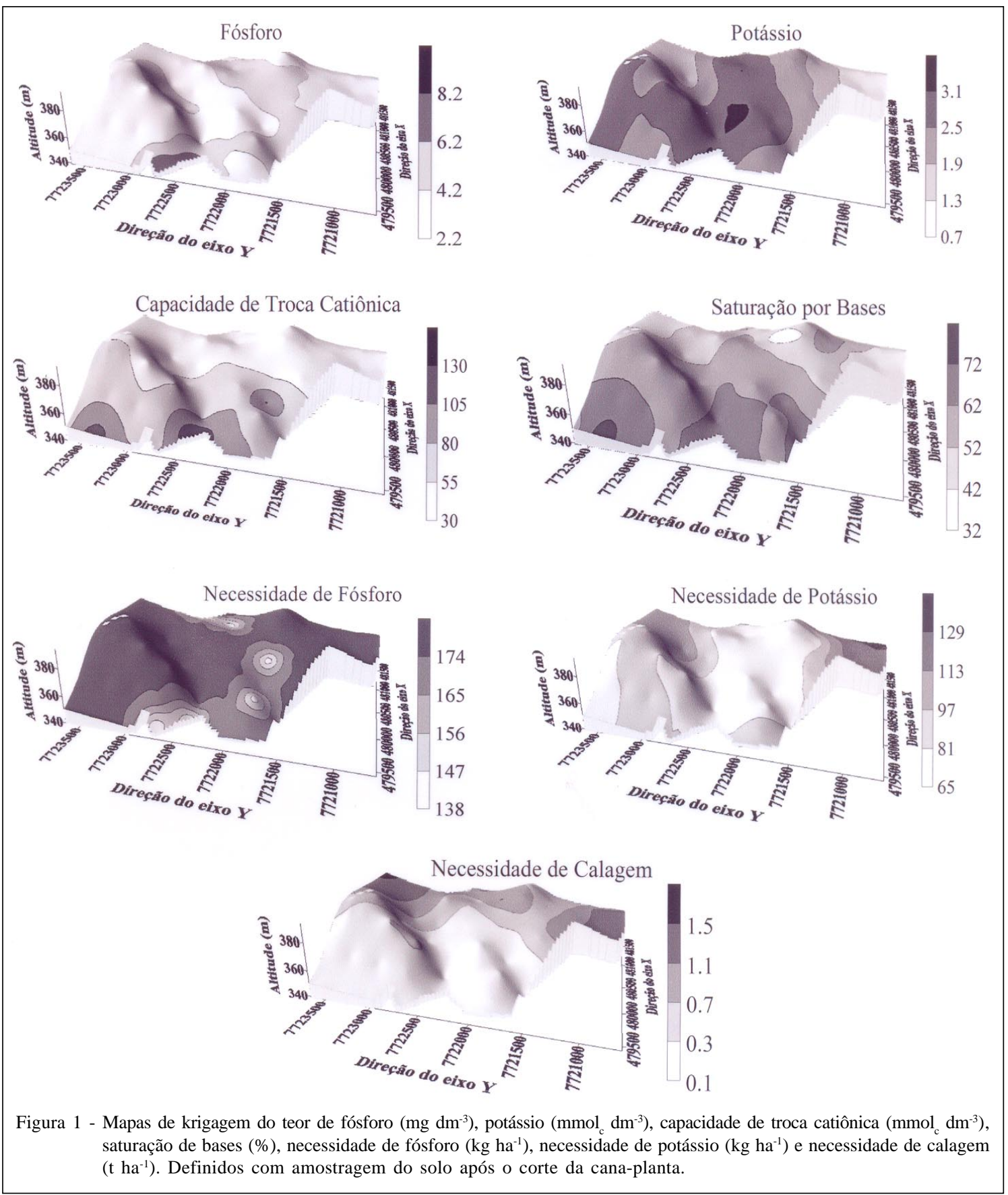

área não necessitando da aplicação de calcário, pois apresenta necessidade abaixo de $1 \mathrm{t} \mathrm{ha}^{-1}$ (Tabela 3 ). Esse fato se deve, provavelmente, devido ao pouco tempo transcorrido da última aplicação de calcário. Segundo RAIJ et al. (1997) e CORÁ \& BERALDO (2006), para valor abaixo de 1,0 t ha $^{-1}$ não se justifica a aplicação de calcário, devido à dificuldade de regulagem dos equipamentos disponíveis no mercado.

O teor médio de fósforo na área foi de 3,90mg $\mathrm{dm}^{-3}$, teor muito baixo segundo RAIJ et al. (1997), portanto, a recomendação média de $\mathrm{P}_{2} \mathrm{O}_{5}$ para área é de $180 \mathrm{~kg} \mathrm{ha}^{-1}$ após o corte da cana-planta (Tabela 1 ). Esse valor baixo de fósforo se deve provavelmente pela localização da adubação no fundo do sulco de plantio. Desse modo, amostragens na entre linha da cultura dificilmente detectarão aumentos nos teores disponíveis do nutriente no primeiro anos após a implantação da cultura. Observa-se que $100 \%$ da área apresentam valores variando de muito baixo a médio para o fósforo (Tabela 3). Estes valores indicam que a 
Tabela 3 - Porcentagem de área de cada classe para os atributos fósforo $\left(\mathrm{mg} \mathrm{dm}^{-3}\right)$, potássio $\left(\mathrm{mmol}_{\mathrm{c}} \mathrm{dm}^{-3}\right)$, capacidade de troca catiônica $\left(\mathrm{mmol}_{\mathrm{c}} \mathrm{dm}^{-3}\right)$, saturação por bases (\%), necessidade de calagem (t ha $\left.{ }^{-1}\right)$, necessidade de fósforo ( $\mathrm{kg}^{-1}$ ) e necessidade de potássio $\left(\mathrm{kg} \mathrm{ha}^{-1}\right)$, em amostragem de solo após o corte da cana-planta.

\begin{tabular}{|c|c|c|c|c|c|c|c|}
\hline \multirow{2}{*}{ Classe } & \multicolumn{7}{|c|}{ Porcentagem de classe (\%) } \\
\hline & Fósforo & Potássio & CTC & V\% & $\mathrm{NC}$ & NP & NK \\
\hline 1 & 40 & 10 & 51 & 4 & 45 & 3 & 45 \\
\hline 2 & 46 & 15 & 35 & 42 & 37 & 2 & 40 \\
\hline 3 & 11 & 25 & 7 & 40 & 9 & 3 & 2 \\
\hline 4 & 3 & 48 & 4 & 12 & 5 & 7 & 7 \\
\hline 5 & - & 2 & 3 & 2 & 4 & 85 & 6 \\
\hline
\end{tabular}

dose de fósforo aplicado na adubação de implantação da cultura não foi suficiente. As doses recomendadas para a aplicação de $\mathrm{P}_{2} \mathrm{O}_{5}$ variam de 138 a $174 \mathrm{~kg} \mathrm{ha}^{-1}$ quando levadadas em conta as particularidades de cada célula da área (Figura 1). Analisando-se o mapa de necessidade de fósforo, verificou-se que $92 \%$ da área (467ha) necessitam de grandes quantidades de adubo fosfatado (Tabela 3). A recomendação média de necessidade de fósforo está muito próxima da recomendação pela taxa variada, portanto neste caso não se recomenda a aplicação de fósforo por taxa variada.

O teor médio de potássio na área foi de 2,18mmol $\mathrm{dm}^{-3}$, considerado como teor médio segundo RAIJ et al. (1997), portanto a recomendação média de $\mathrm{K}_{2} \mathrm{O}$ para área é de $120 \mathrm{~kg} \mathrm{ha}^{-1}$ após o corte da canaplanta (Tabela 1). Observa-se que 98\% da área apresentam valores variando de muito baixo a médio para o potássio e somente $2 \%$ apresentam valores considerados altos (Tabela 3). As doses recomendadas para a aplicação de $\mathrm{K}_{2} \mathrm{O}$, levando em consideração a variação da necessidade de potássio pelo mapa de krigagem, foi de 65 a $129 \mathrm{~kg} \mathrm{ha}^{-1}$ (Figura 1). Analisandose o mapa de necessidade de potássio e a porcentagem de cada classe para o potássio na tabela 3 , verificou-se que $90 \%$ da área (455ha) necessitam de quantidades de potássio abaixo da recomendação média (120 kg ha-1), portanto, para o potássio, é indicada a aplicação em taxa variada. Resultados semelhantes foram observados por WEIRICH NETO et al. (2006).

\section{CONCLUSÃO}

As técnicas de geoestatísticas apresentamse como ferramentas auxiliares na aplicação de fertilizantes por meio de taxa variada, detectando para área em estudo que não se recomenda à aplicação de fósforo e calagem por taxa variada.

\section{REFERÊNCIAS}

BORGELT, S.C. et al. Spatially variable liming rates: a method for determination. Transactions of the ASAE, St. Joseph, v.37, n.5, p.1499-1507, 1994.

BOUMA, J. et al. Pedology, precision agriculture, and the changing paradigm of agricultural research. Soil Science Society of America Journal, Madison, v.63, n.6, p.17631768, 1999.

CAMBARDELLA, C.A. et al. Field-scale variability of soil properties in Central Iowa Soils. Soil Science Society American Journal, Madison, v.58, n.5, p.1501-1511, 1994.

CORÁ, J.E.; BERALDO, J.M.G. Variabilidade espacial de atributos do solo antes e após calagem e fosfatagem em doses variadas na cultura de cana-de-açúcar. Engenharia Agrícola, Jaboticabal, v.26, n.2, p.374-387, 2006.

DERCON, G. et al. Spatial variability in crop response under contour hedgerow systems in the Andes region of Ecuador. Soil \& Tillage Research, Amsterdam, v.86, n.1-2, p.15-26, 2006.

GOLDEN SOFTWARE INC. (Golden, Estados Unidos). Surfer for windows: realese 7.0, contouring and 3D surface mapping for scientist's engineers user's guide. New York, 1999. 619p.

INSTITUTO DE PESQUISAS TECNOLOGICAS DO ESTADO DE SÃO PAULO. Mapa Geológico do Estado de São Paulo. Escala - 1:500.000. São Paulo: IPT, 1981. V.1. 126p.

ISAAKS, E.H.; SRIVASTAVA, R.M. An introduction to applied geoestatistics. New York: Oxford University, 1989. 561p.

JOHNSON, R.M.; RICHARD JUNIOR, E.P. Sugarcane yield, sugarcane quality, and soil variability in Louisiana. Agronomy Journal. Madison, v.97, n.3, p.760-771, 2005.

JUNG W.K. et al. Spatial characteristics of claypan soil properties in an agricultural field. Soil Science Society of American Journal, Madison, v.70, n.4, p.1387-1397, 2006.

MOTOMIYA, A.V.A. et al. Uso da krigagem indicatriz na avaliação de indicadores de fertilidade do solo. Revista Brasileira de Ciência do Solo, Viçosa, v.30, n.3, p.485496, 2006. 
MZUKU, M. et al. Spatial variability of measured soil properties across site-specific management zones. Soil Science Society American Journal, Madison, v.69, n.5, p.1572-1579, 2005.

RAIJ, B. Van et al. Recomendações de adubação e calagem para o estado de São Paulo. 2.ed. Campinas: Instituto Agronômico/Fundação IAC, 1997. 285p. (Boletim Técnico, 100).

RAIJ, B. van et al. Análise química para avaliação da fertilidade de solos tropicais. Campinas: Instituto Agronômico, 2001. 285p.

SCHLINDWEIN, J.A.; ANGHINONI, I. Variabilidade especial de atributos de fertilidade e amostragem de solo no sistema plantio direto. Revista Brasileira de Ciência do Solo, Viçosa, v.24, n.1, p.85-91, 2000.

SCHLOTZHAVER, S.D.; LITTELL, R.C. SAS: system for elementary statistical analysis. 2.ed. Cary, 1997. 905 p.

SPAROVEK, G.; SCHNUG, E. Soil tillage and precision agriculture: A theoretical case study for soil erosion control in
Brazilian sugar cane production. Soil and Tillage Research, Amsterdam, v.61, n.1-2, p.47-54, 2001.

VANNI, S.M. Modelos de regressão: estatística aplicada. São Paulo: Legmar Informática \& Editora, 1998. 177p.

VIEIRA, S.R. et al. Geostatistical theory and application to variability of some agronomical properties. Hilgardia, Berkeley, v.51, n.1, p.1-75, 1983.

WARRICK, A.W.; NIELSEN, D.R. Spatial variability of soil physical properties in the field. In: HILLEL, D. (Ed.). Applications of soil physics. New York: Academic, 1980. Cap.2, p.319-344.

WEIRICH NETO, P.H. et al. Necessidade de fertilizante e calcário em área sob sistema de plantio direto considerando variabilidade espacial. Revista Brasileira de Engenharia Agrícola e Ambiental, Campina Grande, v.10, n.2, p.338343, 2006. 